\title{
Perfil epidemiológico dos casos notificados de intoxicação exógena no Estado do
}

\section{Piauí, Brasil}

\author{
Epidemiological profile of reported cases of exogenous poisoning in the State of Piauí, Brazil \\ Perfil epidemiológico de los casos notificados de intoxicación exógena en el Estado de Piauí, Brasil
}

Recebido: 24/04/2021 | Revisado: 01/05/2021 | Aceito: 02/05/2021 | Publicado: 15/05/2021

João Paulo da Silva-Sampaio

ORCID: https://orcid.org/ 0000-0002-1905-1540 Faculdade Aespi, Brasil

E-mail: joao-sampaio@ hotmail.com

Renan de Lima Costa

ORCID: https://orcid.org/0000-0002-2930-0096

Faculdade Aespi, Brasil

E-mail: renanlima.32@hotmail.com

Kely Naiara dos Santos Torres

ORCID: https://orcid.org/0000-0003-2851-1710

Centro Universitário UNINOVAFAPI, Brasil

E-mail: kellynayarabv@gmail.com

Nataniel Vieira de Sousa

ORCID: https://orcid.org/0000-0002-2107-061X

Centro Universitário UNINOVAFAPI, Brasil

E-mail: natanlove2012@gmail.com

Tatiana Vieira Souza Chaves

ORCID: https://orcid.org/0000-0003-1679-5808

Centro Universitário UNINOVAFAPI, Brasil

E-mail: tatianavsc@yahoo.com.br

Antônio Luiz Gomes Júnior

ORCID: https://orcid.org/0000-0003-0585-3945

Faculdade Aespi, Brasil

E-mail: gomesjunioral@gmail.com

\begin{abstract}
Resumo
Este trabalho teve como objetivo descrever o perfil epidemiológico de intoxicações exógenas notificadas no estado do Piauí - Brasil entre os anos de 2013 a 2017. Foi desenvolvida uma pesquisa de caráter exploratória, epidemiológica e descritiva com abordagem transversal, quantitativo e retrospectivo, os dados foram retirados do Sistema Nacional de Agravos de Notificações (SINAN) do Sistema Único de Saúde (SUS), o perfil evidenciou o predomínio do sexo feminino, faixa etária entre 20-39 anos, ensino fundamental incompleto, o agente tóxico mais frequente foram os medicamentos, as circunstâncias foram as tentativas de suicídios e com evolução de cura sem sequela na maioria dos casos. Foram registrados um total de 6.113 casos de intoxicações entre os anos analisados. Conclui-se, com esse estudo, a importância das notificações como proposta de implementação de melhorias contínuas das notificações e do preenchimento das fichas no SINAN, pois nos permitirá a obtenção de dados confiáveis e consistentes.
\end{abstract}

Palavras-chave: Intoxicações exógenas; Notificações; Perfil epidemiológico.

\begin{abstract}
This study aimed to describe the epidemiological profile of exogenous intoxications reported in the state of Piauí Brazil between the years 2013 to 2017. An exploratory, epidemiological and descriptive research was carried out with a cross-sectional, quantitative and retrospective approach, the data were taken from of the National System of Notifiable Diseases (SINAN) of the Unified Health System (SUS), the profile showed a predominance of females, aged between 20-39 years old, incomplete elementary school, the most frequent toxic agent were medicines, circumstances were suicide attempts and evolution with no sequel in most cases. A total of 6,113 cases of poisoning were recorded between the years analyzed. We conclude, with this study, the importance of notifications as a proposal for the implementation of continuous improvements of notifications and the filling of the forms in SINAN, as it will allow us to obtain reliable and consistent data.
\end{abstract}

Keywords: Exogenous intoxications; Notifications; Epidemiological profile. 


\begin{abstract}
Resumen
Este estudio tuvo como objetivo describir el perfil epidemiológico de intoxicaciones exógenas reportadas en el estado de Piauí - Brasil entre los años 2013 a 2017. Se realizó una investigación exploratoria, epidemiológica y descriptiva con un enfoque transversal, cuantitativo y retrospectivo, los datos fueron tomado del Sistema Nacional de Enfermedades Notificables (SINAN) del Sistema Único de Salud (SUS), el perfil mostró un predominio del sexo femenino, con edades entre 20-39 años, primaria incompleta, el tóxico más frecuente fueron los medicamentos, circunstancias fueron intentos de suicidio y evolución sin secuela en la mayoría de los casos. Se registraron un total de 6.113 casos de intoxicación entre los años analizados. Concluimos, con este estudio, la importancia de las notificaciones como propuesta para la implementación de mejoras continuas de notificaciones y el llenado de formularios en SINAN, ya que nos permitirá obtener datos confiables y consistentes.
\end{abstract}

Palabras clave: Intoxicaciones exógenas; Notificaciones; Perfil epidemiológico.

\title{
1. Introdução
}

A Intoxicação é a manifestação que ocorre por meio de sinais e sintomas, dosefeitos desfavoráveis produzidos em um organismo vivo a partir da interação com o contato com agente tóxico externo. São destacadas como principais agentes que causam intoxicação as seguintes substâncias: produtos químicos, plantas tóxicas, agrotóxicos agrícolas, drogas, cosméticos, medicamentos e alimentos (Ramos, et al., 2017; Silva \& Costa, 2018).

Dessa forma, a intoxicação crônica manifesta-se por meio de várias patologias, que atingem inúmeros órgãos e sistemas, com ênfase para os problemas imunológicos, hematológicos, hepáticos, neurológicos, malformações congênitas e tumores. Diante disso, as intoxicações crônicas através dos agrotóxicos encontram- se mencionadas em vários capítulos da Classificação Internacional de Doenças. Nesse interim, os acontecimentos de intoxicações por agrotóxicos mostram um crescimento nas ocorrências de tentativas de suicídio e contaminação de trabalhadores rurais pelo uso inadequado de agrotóxicos agrícolas, ocasionando sequelas, algumas vezes irreversíveis, danos ao sistema nervoso central, efeitos mutagênicos, carcinogênicos e teratogênicos, mais adiante uma série de outras doenças e complicações de saúde (Costa \& Rohlfs, 2011).

Além disso, a Notificação tem uma função de planejar estratégias para incentivar as ações de prevenção e proteção, sendo uma das ferramentas definidas pelas políticas públicas específicas e está assegurada na legislação Brasileira, passando a ser um instrumento da garantia de direitos e de proteção social (Brasil, 2012).

A Vigilância Epidemiológica é estabelecida pela Lei $\mathrm{n}^{\circ}$ 8.080/90 como um anexo de operações que proporciona o conhecimento, a detecção ou prevenção de qualquer outra alteração nos aspectos determinantes e condicionantes de saúde pública ou individual, com o propósito de sugerir e realizar as providências para a prevenção e controle das doenças ou agravos (Suvisa, 2018).

No Brasil a intoxicação exógena encontra-se entre os três principais meios empregados nas tentativas e suicídios, registrado pela Vigilância de Violências e Acidentes (VIVA), através da Ficha de Notificação/Investigação de Violência Doméstica, Sexual e/ou outras Violências (Brasil, 2014; Santos, et al., 2013).

Neste contexto, este estudo tem o objetivo de identificar a prevalência dos casos de intoxicação exógena, no Piauí, entre os anos de 2013 a 2017, assunto este, de interesse acadêmico, social, econômico e de saúde pública, pois são substâncias que causam problemas para a saúde das pessoas que sofreram intoxicações por acidentes, intoxicação por alimento, exposição prolongada aos agrotóxicos, intoxicação medicamentosa, ou por tentativa de suicídio utilizando essas substâncias,sendo uma das causas que representa riscos para a saúde humana, podendo levar amorte do indivíduo. 


\section{Metodologia}

Trata-se de um estudo ecológico transversal, de abordagem quantitativa, e caráter exploratório-descritivo, para traçar os casos de Intoxicação Exógena registrados no Piauí. A pesquisa quantitativa segue o método baseado na mensuração, análise e controle rigoroso de dados numéricos e quantificáveis (Pereira, et al., 2018).

O cenário do estudo é o estado do Piauí, situado na região nordeste do Brasil, entre2 $2^{\circ} 44^{\prime} 49^{\prime \prime}$ e $10^{\circ} 55^{\prime} 05^{\prime \prime}$ de latitude sul e $40^{\circ} 22^{\prime} 12^{\prime \prime}$ e $45^{\circ} 59^{\prime} 42^{\prime \prime}$ de longitude oeste, apresentando um total de 224 municípios e, aproximadamente, 3.118.360 habitantes. Constitui-se em uma região de clima semiárido, apresentando escassez e irregularidadesde chuvas, com precipitações entre $600 \mathrm{~mm}$ e $1200 \mathrm{~mm}$ e temperaturas elevadas, comlongo período de seca (Brasil, 2009; Silva, et al., 2010).

Os dados de registros da intoxicação exógena foram coletados entre os meses de março e abril do ano de 2020. A coleta de dados procedeu-se através de buscas no Sistema de Informação de Agravos de Notificação (SINAN) do Departamento de Informática do Sistema Único de Saúde (DATASUS).

Dessa forma, foi acessado o site do SINAN (www2. datasus.gov.br/DATASUS), no qual realizou-se os seguintes passos: clicou-se em dados epidemiológicos e morbidades, localizado no lado esquerdo da página; selecionou-se doenças e agravos de notificação; período de 2007 em diante; prosseguiu-se clicando no tópico intoxicação exógena e na imagem que representa o território do Piauí. Posteriormente, foi selecionada Intoxicação exógena, buscando na base de dados (SINAN) os dadosalimentados entre 2013 e 2017, utilizando-se os critérios do objetivo específico.

Após a coleta de dados, foram criadas planilhas para o estudo das variáveis relativas aos casos de intoxicações exógenas no Piauí, sendo estes: agentes tóxicos, faixa etária, nível de escolaridade, sexo, circunstâncias e evolução no período de 2013 a 2017.

A interpretação das informações foi realizada de acordo com as estatísticas apresentadas para cada uma das variáveis relevantes para o tema em análise, buscando-se as possíveis hipóteses para os achados embasadas na literatura científica, acerca das intoxicações exógenas. Algumas limitações foram encontradas durante a análise devido ao fato que algumas variáveis foram ignoradas e/ou não informadas.

\section{Resultados e Discussão}

As intoxicações exógenas são consideradas um problema de saúde pública com casos em todo o mundo devido à diversidade do espaço geográfico com perfis diferentes. Durante o período de 2013 a 2017, no estado do Piauí, foi notificado um total de 6.113 casos de intoxicações exógenas, uma média de 1.222 casos entre os anos analisados. $\mathrm{O}$ ano de maior ocorrência desse tipo de incidência foi 2017 com 1.580 casos e o de menor número de notificação foi 2013 com 872 casos de intoxicações. As Tabelas abaixo demonstram casos notificados e confirmados de intoxicaçãoexógena no Piauí entre os anos de 2013 a 2017 de acordo com as seguintes variáveis: sexo, agente tóxico, evolução, ano de notificação, circunstância, faixa etária e escolaridade.

Observou-se um aumento significativo no número de casos registrados pelo sexo feminino atingindo 1.001 notificações no ano de 2017. Entre os anos analisadoso sexo masculino apresentou menor número de casos de notificações em relação aosexo feminino que disparou com uma soma de 3.704 de casos notificados, mais de $60 \%$ do total geral do número de intoxicações, conforme dados expostos na Tabela 1. 
Tabela 1. Intoxicação exógena segundo sexo dos casos notificados no estado do Piauí entreo período de 2013 a 2017.

\begin{tabular}{lccccccc}
\hline & \multicolumn{9}{c}{ Ano de notificação } \\
\cline { 2 - 8 } SEXO & 2013 & 2014 & 2015 & 2016 & 2017 & Total & $\%$ \\
\hline Masculino & 331 & 458 & 425 & 616 & 579 & 2409 & 39,4 \\
Feminino & 541 & 614 & 712 & 836 & 1001 & 3704 & 60,6 \\
\hline
\end{tabular}

Fonte: Ministério Saúde/SVS - Sistema de Informação de Agravos de notificação. - Sinan. Net.

De acordo com Vieira, Santana e Suchara (2015) fatores como agressões domésticas ocasionadas pelo parceiro, maior exposição ao abuso sexual na infância, fragilidade frente a fatores psicológicos e sociais estressantes, formação de patologiasmentais, além de estar sob influência de uma cultura associada à igualdade de gênerosão alguns fatores que podem deixar mulheres mais expostas e vulneráveis as tentavas de suicídio, ou seja, tendo em vista as condições sociais das quais as mulheres estão inseridas pode levar a um maior número de intoxicações exógenas pelo sexo feminino (Veloso, et al., 2017).

Segundo Vijayakumar et., al (2017) a automedicação e a susceptibilidade paratentativas de suicídio são favorecidas pela compra de medicamentos que são mantidos na residência. A maior procura pelos serviços de saúde e o maior uso de medicamentos, que levariam ao acesso fácil ao agente intoxicante, justificariam o porquê das mulheres serem o sexo mais predominante nos casos de intoxicações exógenas por tentativas de suicídios.

Foi observado que houve um aumento gradativo das intoxicações por medicamentos registrando 889 pessoas no ano de 2017, ficando em segundo lugar os alimentos e bebidas apresentando 453 casos, totalizando 6.113 notificações pelo SINAN entre os anos de 2013 a 2017.

Em relação à evolução de intoxicações exógenas os casos de curas sem sequelas foram totalizados 4.628, cerca de $75,7 \%$ das notificações. Em relação aos anos de notificação mostra que ocorreu um aumentode acordo com os anos entre 2013 a 2017 respectivamente, ficando com maior número de casos no ano de 2017 com 1.580, totalizando 6113 notificações durante esses anos, de acordo com a Tabela 2. 
Tabela 2. Intoxicação exógena segundo agente tóxico, evolução, e circunstanciados casosnotificados no estado do Piauí entre o período de 2013 a 2017.

\begin{tabular}{|c|c|c|c|c|c|c|c|}
\hline \multirow[b]{3}{*}{ AGENTE TÓXICO } & \multicolumn{5}{|c|}{ Ano de notificação } & \multirow{3}{*}{ Total } & \multirow{3}{*}{$\%$} \\
\hline & 2013 & 2014 & 2015 & 2016 & 2017 & & \\
\hline & & & & & & & \\
\hline Medicamento & 444 & 524 & 628 & 760 & 889 & 3245 & 53.08 \\
\hline Agrotóxico agrícola & 22 & 22 & 23 & 39 & 44 & 150 & 2.45 \\
\hline Agrotóxico doméstico & 23 & 16 & 13 & 20 & 24 & 96 & 1.57 \\
\hline Agrotóxico saúde pública & 4 & 3 & 2 & 3 & - & 12 & 0.19 \\
\hline Raticida & 44 & 48 & 47 & 65 & 58 & 262 & 4.28 \\
\hline Prod. Veterinário & 13 & 18 & 12 & 19 & 17 & 79 & 1.29 \\
\hline Prod. uso domiciliar & 41 & 80 & 79 & 108 & 83 & 391 & 6.39 \\
\hline Cosmético & 11 & 21 & 26 & 29 & 24 & 111 & 1.81 \\
\hline Prod. Químico & 28 & 43 & 34 & 36 & 44 & 185 & 3.02 \\
\hline Metal & - & 1 & - & - & - & 1 & 0.01 \\
\hline Drogas de abuso & 15 & 16 & 20 & 53 & 88 & 192 & 3.14 \\
\hline Planta tóxica & 14 & 8 & 10 & 15 & 17 & 64 & 1.04 \\
\hline Alimento e bebida & 54 & 76 & 75 & 145 & 103 & 453 & 7.41 \\
\hline Outro & 16 & 20 & 18 & 53 & 96 & 203 & 3.32 \\
\hline Ign/branco & 143 & 176 & 150 & 107 & 93 & 669 & 10.9 \\
\hline \multicolumn{8}{|l|}{ EVOLUÇÃO } \\
\hline Cura sem sequela & 689 & 871 & 938 & 1168 & 962 & 4628 & 75.7 \\
\hline Cura com sequela & 8 & 12 & 12 & 13 & 13 & 58 & 0.94 \\
\hline Óbito por intoxicação & 8 & 14 & 15 & 15 & 8 & 60 & 0.98 \\
\hline Óbito por outra causa & - & 1 & - & 1 & 2 & 4 & 0.06 \\
\hline Perda de Seguimento & 4 & 3 & 14 & 12 & 14 & 47 & 0.76 \\
\hline Ign/branco & 163 & 171 & 158 & 243 & 581 & 1316 & 21.5 \\
\hline \multicolumn{8}{|l|}{ CIRCUNSTÂNCIA } \\
\hline Uso habitual & 45 & 93 & 63 & 169 & 62 & 432 & 7.06 \\
\hline Acidental & 208 & 242 & 207 & 254 & 250 & 1161 & 18.9 \\
\hline Ambiental & 6 & 11 & 6 & 5 & 5 & 33 & 0.53 \\
\hline Uso terapêutico & 34 & 85 & 73 & 115 & 126 & 433 & 7.08 \\
\hline Prescrição médica & 1 & 2 & 1 & 2 & 2 & 8 & 0.13 \\
\hline Erro de administração & 20 & 14 & 20 & 18 & 15 & 87 & 1.42 \\
\hline Automedicação & 32 & 38 & 45 & 46 & 45 & 206 & 3.36 \\
\hline Abuso & 23 & 16 & 43 & 57 & 86 & 225 & 3.68 \\
\hline Ingestão de alimento & 44 & 47 & 59 & 55 & 70 & 275 & 4.49 \\
\hline Tentativa de suicídio & 308 & 347 & 446 & 524 & 680 & 2305 & 37.7 \\
\hline Tentativa de aborto & 3 & 3 & 1 & 2 & 3 & 12 & 0.19 \\
\hline Violência/Homicídio & 4 & 6 & 15 & 76 & 110 & 211 & 3.45 \\
\hline Outra & 8 & 10 & 12 & 14 & 13 & 57 & 0.93 \\
\hline Ign/ branco & 136 & 158 & 146 & 115 & 113 & 668 & 10.9 \\
\hline
\end{tabular}

Fonte: Ministério Saúde/SVS - Sistema de Informação de Agravos de notificação. - Sinan. Net.

Os medicamentos são agentes tóxicos que proporcionam intoxicações acidentais por vários fatores, sendo a automedicação uma das práticas mais adotadas pela população. Porem, esta não é a única responsável, pois há casos que ocorrem erros na prescrição médica em relação à dosagem, ou até mesmo na medicação (Carvalho, et al., 2017). Ressalta-se também a associação com outros medicamentos tóxicos, como drogas abusivas que proporciona uma intoxicação maispotente e comprometedora para o organismo (Mathias et al., 2019).

É crescente a utilização de medicamentos, principalmente os relacionados a doenças psicológicas, nas tentativas de suicídios, cujas causas podem ser diversas, desde culturais ou falhas na precisão e dispensação (Carvalho et al., 2017). 
Segundo o autor Nunes et al., 2017, afirma que as intoxicações medicamentosas ocorrem como principal circunstância as tentativas de suicídio nos jovens adultos e dosexo feminino corroborando com os dados do presente estudo.

Nas intoxicações alimentares a maior parte é relacionada ao consumo de alimentos incluindo bactérias patogênicas ou toxinas. As condições higiênicas doslocais de produção e manipulação dos alimentos interferem na qualidade microbiológica por serem considerados sinais de contaminação e os manipuladores são constantemente disseminadores de agentes patogênicos (Passos, et al., 2010).

As intoxicações alimentares podem ser determinadas como todas as doenças de natureza infecciosa ou tóxica, provocada pelo consumo de alimentos ou bebidas contaminadas com agentes patogênicos ou suas toxinas. Dentre esses patógenos, destacam-se os fungos dos gêneros de Aspergillus, Fusarium e Penicillium. Quanto à intoxicação alimentar as bactérias que mais se destacam, por exemplo: Shigella, Clostridium, Salmonella, Staphylococcus e Bacillus (Almeida et al., 2008; Martinovic et al., 2016).

Em relação às circunstâncias das intoxicações foram notificados $37,7 \%$ que estavam relacionados à tentativa de suicídio e 18,9\% a causas acidentais. Osmedicamentos são os que representam o maior número de tentativas de suicídio em que se observa um real crescimento na utilização para esta finalidade, pode haver vários fatores, desde culturais até falhas na prescrição e dispensação (Carvalho, et al., 2017). Segundo Nunes et al. 2017, a tentativa de suicídio foi a principal circunstância relacionada às intoxicações medicamentosas nos jovens adultos, principalmente nas mulheres, que apresentam uma frequência duas vezes mais elevada de tentativa de suicídio em relação aos homens.

Os dados da Tabela 2 descrevem também o rastreamento da evolução dos casos de intoxicação exógena por prevalência de casos. Neste parâmetro, notou-se um total de cura sem sequela de 4.628 casos notificados (75,7\%), em 2016 foram registrados 1.168 casos e no ano de 2017 houve uma redução com 962 casos. Foram registrados 60 óbitos durante o período de 2013 a 2017. Em estudo realizado por Silva Filho (2009) e Da Silva et., al (2010) a cura sem sequela constitui-se no tipo de evolução das intoxicações mais constante, com 95,8\% e 80\% do total de casos, respectivamente. Esse percentual demostra de forma geral que os atendimentos hospitalares estão correspondendo às ocorrências adequadamente, mesmo sem serviço especializado.

A distribuição da frequência relativa das intoxicações exógenas, notificadas por faixa etária no período de 2013 a 2017, demonstrou que as faixas etárias de maior ocorrência estão entre as idades de 20 a 39 anos, com registros de 2.381 casos eentre 40 a 59 anos, com 936 casos notificados no Piauí pelo SINAN no período de 2013 a 2017, como se expõe na Tabela 3. 
Tabela 3. Intoxicação exógena segundo faixa etária e escolaridade dos casos notificados noestado do Piauí entre o período de 2013 a 2017.

\begin{tabular}{|c|c|c|c|c|c|c|c|}
\hline & \multicolumn{5}{|c|}{ Ano de notificação } & \multirow[b]{2}{*}{ Total } & \multirow[b]{2}{*}{$\%$} \\
\hline & 2013 & 2014 & 2015 & 2016 & 2017 & & \\
\hline \multicolumn{8}{|l|}{ FAIXA ETÁRIA } \\
\hline$<1$ Ano & 27 & 30 & 34 & 43 & 38 & 172 & 2.81 \\
\hline $1-4$ & 144 & 178 & 151 & 211 & 200 & 884 & 14.4 \\
\hline $5-9$ & 53 & 69 & 46 & 72 & 72 & 312 & 5.10 \\
\hline $10-14$ & 65 & 62 & 55 & 79 & 96 & 357 & 5.84 \\
\hline $15-19$ & 121 & 119 & 153 & 163 & 224 & 780 & 12.7 \\
\hline $20-39$ & 297 & 388 & 453 & 605 & 638 & 2381 & 38.9 \\
\hline $40-59$ & 130 & 165 & 195 & 207 & 239 & 936 & 15.3 \\
\hline $60-64$ & 10 & 19 & 15 & 26 & 30 & 100 & 1.63 \\
\hline $65-69$ & 13 & 9 & 15 & 17 & 14 & 68 & 1.11 \\
\hline $70-79$ & 8 & 19 & 13 & 20 & 16 & 76 & 1.24 \\
\hline $80 \mathrm{e}+$ & 4 & 14 & 7 & 9 & 13 & 47 & 0.76 \\
\hline \multicolumn{8}{|l|}{ ESCOLARIDADE } \\
\hline Analfabeto & 19 & 10 & 15 & 8 & 14 & 66 & 1.07 \\
\hline Fundamental incompleto & 147 & 151 & 136 & 170 & 196 & 800 & 13.0 \\
\hline Fundamental completo & 20 & 17 & 24 & 36 & 30 & 127 & 2.07 \\
\hline Médio incompleto & 48 & 49 & 49 & 52 & 90 & 288 & 4.71 \\
\hline Médio completo & 54 & 50 & 71 & 86 & 114 & 375 & 6.13 \\
\hline Superior incompleto & 16 & 14 & 34 & 23 & 30 & 117 & 1.91 \\
\hline Superior completo & 5 & 14 & 15 & 25 & 33 & 92 & 1.50 \\
\hline Ign/Branco & 563 & 767 & 793 & 1052 & 1073 & 4248 & 69.4 \\
\hline
\end{tabular}

Fonte: Ministério Saúde/SVS - Sistema de Informação de Agravos de notificação. - Sinan. Net.

O grande número de doenças crônicas presente, principalmente na populaçãojovem adulta, desencadeia inúmeras pesquisas para elaboração e produção de novos fármacos. Esse grande quantitativo de medicamentos disponível no mercado favorece o aumento de prescrições médicas, que, muitas vezes, são desnecessárias e podem causar efeitos colaterais no paciente, interações medicamentosas, reações adversas graves, riscos de iatrogênicas, hospitalizações e até a morte do indivíduo (Pereira, et al., 2017).

Em relação à faixa etária de 20-39 anos o uso abusivo de medicamentos podeser decorrente ainda da tentativa de provocar sua morte, o que expõe a necessidade de implantar programas de assistência que permitam identificar situações de risco para o ato suicida (Carvalho, et al 2017).

Em relação à escolaridade, verificou-se um maior número de casos, 800pessoas $(13,0 \%)$ com ensino fundamental incompleto. Em seguida, obteve-se 375 casos $(6,13 \%)$ de pessoas que possuíam o ensino médio completo. O baixo nível de escolaridade apresenta-se como um fator de risco para a realização de violência autoprovocada por intoxicação exógena. A falta de escolarização está, geralmente, relacionada a dificuldades socioeconômicas, podendo resultar em grandes prejuízos à qualidade de vida individual e familiar e, consequentemente, aumentar o risco de comportamentos suicidas (Vieira, et. al., 2015). Ainda em relação ao critério "grau deescolaridade", vale salientar que para os trabalhadores que manipulam produtos, como no caso dos agrotóxicos, o baixo nível de escolaridade pode dificultar o acesso a informações importantes para sua segurança na atividade laboral, sendo necessárias diferentes ações de saúde pública e ocupacional para diminuição do riscode intoxicação (Parra, et al., 2017). 


\section{Conclusão}

Os resultados do presente estudo demonstram que as intoxicações exógenas no Piauí atingiram significativamente um perfil jovem (20 - 39 anos), com maior incidência no sexo feminino, sendo os medicamentos o principal agente tóxico utilizado, em tentativas de suicídios.

Nesse sentido, evidencia-se que as notificações possibilitam traçar um perfil mais apurado das ocorrências, que serve de parâmetro para o direcionamento das políticas de promoção e assistência à saúde, assim como, o planejamento de ações para problemas específicos e, muitas vezes, menos visíveis aos olhos dos gestores, dos profissionais de saúde e de toda a sociedade, que afetam adultos jovens, como o suicídio, cujas tentativas mesmo sem sequelas físicas podem acarretar traumas, dificuldades de sociabilidade e transtornos ao paciente e a toda sua família. Assim, novos estudos sobre a temática deverão acontecer para acompanhar a evolução do perfil das intoxicações exógenas no estado do Piauí.

\section{Referências}

Almeida, C. F., Araújo, E. S., Soares, Y. C., Diniz, R. L. C., Fook, S. M. L., \& Viera, K. V. M. (2008). Perfil epidemiológico das intoxicações alimentares notificadas noCentro de Atendimento Toxicológico de Campina Grande, Paraíba. Revista Brasileira Epidemiologia, 11(1).

Brasil. (2014). Ministério da Saúde. Secretaria de Vigilância em Saúde. Instrutivo para preenchimento da ficha de notificação de Violência interpessoal/autoprovocada.

Brasil. (2012). Ministério da Saúde. Indicadores e Dados Básicos. Indicadores de morbidade. Taxa de incidência da leishmaniose visceral.

Brasil. (2009). Ministério da Saúde. Sistema Nacional de Vigilância em Saúde.Relatório de Situação: Piauí.

Carvalho, F. S. A., Mororó, W. M. V., Alencar, Y. C. A., Sette, R. B. T. \& Sousa, M. N. A. (2017). Intoxicação exógena no estado de Minas Gerais, Brasil. Ciência \& Desenvolvimento-Revista Eletrônica da FAINOR. 10(1).

Costa, F. L. F., \& Rohlfs, D. B. (2011). Resíduos de agrotóxicos em alimentos implicaçõespara saúde e meio ambiente. Pontifícia Universidade Católica de Goiás. Programade Pós Graduação em Biociências Forenses. 2011.

Martinović, T., Andjelković, U., Gajdošik, M. S., Rešetar, D., \& Josić, D. (2016). Food borne pathogensand their toxins. Journal of Proteomics. 147(2).

Mastroianni, P. C., Lucchetta, R. C., Sarra, J. R., \& Galduróz, J. C. F. (2011). Estoque doméstico e uso de medicamentos em uma população cadastrada na estratégia saúde da família no Brasil. Revista PanamericanaSalud Publica. 29(5).

Mathias, T. L., Guidoni, C. M., Girotto, E. (2019). Tendências de eventos toxicológicos relacionados a medicamentos atendidos por um Centro de InformaçõesToxicológicas. Revista Brasileira de epidemiologia, 22(3).

Nunes, C. R. M., Alencar, G. O., Bezerra, C. A., Barreto, M. F. R., \& Saraiva, E. M. S. (2017) Panoramas Das Intoxicações Por Medicamentos No Brasil. Revista E-Ciência, 5(2).

Parra, M. L., Pelazza, B. B., Nascimento, N. E., Paula, C. R., \& Silva, J. F. (2017). Saúde do Trabalhador e Intoxicação por Organofosforados: Um Estudo Sistemático. Revista Dialogando Saberes, 1(6).

Passos, E. D. C., Mello, A. R. P. D., Sousa, C. V. D., Silva, C. R. D., Alonso, A. C. B., Gonzalez, E., \& Tavares, M. (2010). Provável surto de toxinfecção alimentar em funcionários de uma empresa no litoral da região sudeste do Brasil. Revista do Instituto Adolfo Lutz, 69(1).

Pereira A. S. et al. (2018). Metodologia da pesquisa científica. [free e-book]. Santa Maria/RS. Ed. UAB/NTE/UFSM. 5.2) Estrela, C. (2018)

Pereira, K. G., Peres, M. A., Iop, D., Boingi, A. C., Boing, A. F., Azizi, M., \& D’orsii, E. (2017). Polifarmácia em Idosos: Um Estudo de Base Populacional. Revista Brasileira Epidemiologia. 20 (2).

Ramos, T. O., Colli, V. C., \& Sanches, A. C. S. (2017). Indicadores epidemiológicos dasintoxicações exógenas em crianças menores de 5 anos na região de Araçatuba- SP. Revista intertox de toxicologia, risco ambiental e sociedade, 10(3).

Santos, S. A., Legay, L. F., Aguiar, F. P., Lovisi, G. M., Abelha, L., \& Oliveira, S. P. (2013). Suicídios e tentativas de suicídios por intoxicação exógena no Rio de Janeiro: análise dos dados dos sistemas oficiais de informação em saúde, 2006-2008. Revista Brasileira de Epidemiologia.16(1).

Silva, C. C. S., Souza, K. S., \& Marques, M. F. L. (2011). Intoxicações Exógenas: Perfil doscasos que necessitaram de assistência intensiva em 2007. Revista Brasileira de Ciencia da Saúde. 15(1).

Silva, H. C. G., \& Costa, J. B. (2018). Intoxicação exógena: casos no estado de Santa Catarina no período de 2011 a 2015 . Arquivos Catarinenses de Medicina, 47(3).

Suvisa, Superintendência de Vigilância em Saúde. Gerência de VigilânciaEpidemiológica (GVE). Estado de Goiás. 2018. 
Research, Society and Development, v. 10, n. 5, e52810515425, 2021

(CC BY 4.0) | ISSN 2525-3409 | DOI: http://dx.doi.org/10.33448/rsd-v10i5.15425

Silva, J.B., Xavier, D. S., Barboza, M. C. N., Amestoy, S. C., Trindade, L. L., \& Silva, J. R. S. (2013). Fumicultores da zona rural de Pelotas (RS), no Brasil:exposiçãoocupacional e a utilização de equipamentos deproteção individual (EPI). Saúde emDebate, 37(97).

Silva, C. M. S., Lima, E. S., Cantalice, M. L., Alencar, M. T., \& Da Silva, W. A. L. (2010). Seminário Piauiense: Educação e Contexto. Campina Grande: Triunfal Gráfica e Editora. (2010).

Veloso, C., Monteiro, C. F. D. S., Veloso, L. U. P., Figueiredo, M. D. L. F., Fonseca, R. S. B., Araújo, T. M. E. D., \& Machado, R. D. S. (2017). Violência autoinfligida por intoxicação exógena em um serviço de urgência e emergência. Revista Gaúcha deenfermagem, 38(2).

Vieira, L. P., Santana, V. T. P., \& Suchara, E. A. (2015) Caracterização de tentativas desuicídios por substâncias exógenas. CadernosSaúdeColetiva, 23(2).

Vijayakumar, Lakshmiet et al. (2017) Challenges and opportunities in suicide prevention in South-East Asia. WHO South-East Asia journal of public health, 6(1). 